\title{
PATH MAPS AND CONTINUITY ${ }^{1}$
}

\section{ERIC BEDFORD}

A function $f: X \rightarrow Y$ will be called a path map if it maps the paths of $X$ to paths of $Y$. That is, if $\sigma: I \rightarrow X$ is a path, then so is $f \sigma: I \rightarrow Y$. Any continuous function is a path map. Conversely, I shall show that with mild restrictions on the space $X$, all path maps $f: X \rightarrow Y$ are continuous.

A duality consideration yields some more information about the situation. In this case, the object of interest is the following: $g: X \rightarrow Y$ is a reverse path map if whenever $\phi: Y \rightarrow I$ is a continuous (real-valued) function, then so is $\phi g: X \rightarrow I$. If certain conditions are placed upon $Y$, then every reverse path map $g: X \rightarrow Y$ is continuous. The aim of the following theorems is to reveal some of the interplay between paths, continuous functions, and continuous real-valued functions.

THEOREM 1. If $X$ is locally path connected, Hausdorff and 1 st countable, then every path map $f: X \rightarrow Y$ is continuous.

Proof. Let $\left\{x_{n}\right\}$ be any sequence in $X$ converging to $x_{0}$. We shall construct a path $\Gamma[0,1] \rightarrow X$ such that $\Gamma(1 / n)=x_{n}, \Gamma(0)=x_{0}$. Let $U_{1} \supseteq U_{2} \supseteq \ldots$ be a $n b$. basis at $x_{0}$ with $U_{n}$ path-connected. We may assume $x_{n} \in U_{1}, n=1,2, \ldots$. For each $n \geqq 1$, let $j_{n}$ be the largest integer such that $x_{n}$ and $x_{n+1}$ lie in $U_{j_{n}}$. Pick a path $\gamma_{n}:[1 /(n+1), 1 / n]$ $\rightarrow U_{j_{n}}$ such that $\gamma_{n}(1 /(n+1))=x_{n+1}, \gamma_{n}(1 / n)=x_{n}$. Now we define $\Gamma:[0,1] \rightarrow X$ as:

$$
\Gamma(0)=x_{0}, \quad \Gamma(t)=\gamma_{n}(t) \quad \text { if } t \in[1 /(n+1), 1 / n] .
$$

Clearly $\Gamma:(0,1] \rightarrow X$ is continuous. To see that $\Gamma$ is continuous at 0 , let $V$ be any $n b$. of $x_{0}$. Then for some integer $K, U_{j} \subseteq V$ whenever $j \geqq K$. Let $N$ be large enough that if $n \geqq N$, then $x_{n} \in U_{K}$. Such an $N$ exists because $\left\{x_{n}\right\}$ converges to $x_{0}$. Further, this means that if $j \geqq N$, then the path $\gamma_{j}$ lies inside of $U_{K}$. Hence $\Gamma^{-1}([0,1 / N]) \subseteq U_{K}$ $\subseteq V$, and thus $\Gamma$ is continuous.

Since $f$ is a path map, $f \Gamma$ will be a path in $Y$ such that $f \Gamma(1 / n)=f\left(x_{n}\right)$ and $f \Gamma(0)=f\left(x_{0}\right)$. Thus $f\left(x_{n}\right)$ converges to $f\left(x_{0}\right)$, and $f$ is continuous. Q.E.D.

Received by the editors May 26, 1969.

1 The author gratefully acknowledges many helpful comments from the referee, especially his correction of the proof of Theorem 1. 
Theorem 2. If $X$ and $Y$ are locally path connected and Hausdorff and 1st countable, and if $f: X \rightarrow Y$ is any (set theoretic) function, then the following are equivalent:

(i) $f$ is a homeomorphism,

(ii) $f^{-1}$ exists, and both $f$ and $f^{-1}$ are path maps,

(iii) $f$ is a path map and the induced $\hat{f}(i . e ., \hat{f}(\sigma)=f \sigma)$ is a set theoretic isomorphism of the sets $X^{I}$ and $Y^{I}$,

(iv) $f$ is a path map and the induced $\hat{f}$ is a homeomorphism in the compact-open topology.

Proof. This is an immediate consequence of Theorem 1. The maps $\hat{f}$ that are induced from path maps $X \stackrel{f}{\rightarrow} Y$ can be described easily.

Theorem 3. If $G: X^{I} \rightarrow Y^{I}$ is a set-theoretic map, and $G$ is invariant under parametrization (i.e., if $\rho: I \rightarrow I$ is a continuous map, then $G(\sigma) \rho=G(\sigma \rho))$, then there is a path map $f: X \rightarrow Y$ such that $\hat{f}=G$.

Proof. If $\tau_{x_{0}}$ is the trivial path at $x_{0}$, then $G\left(\tau_{x_{0}}\right)$ is a trivial path in $Y$. Let $\rho_{t_{0}}: I \rightarrow I$ be the constant map into $t_{0}$. Then $G\left(\tau_{x_{0}}\right)=G\left(\tau_{x_{0}} \rho_{t_{0}}\right)$ $=G\left(\tau_{x_{0}}\right) \rho_{t_{0}}$, and hence $G\left(\tau_{x_{0}}\right)$ is trivial.

Now we define a map: $f: X \rightarrow Y$ as follows. If $x \in X$, and $\tau_{x}$ is the constant path at $x$, then there is a $y \in Y$ such that $G\left(\tau_{x}\right)=\tau_{y}$. We define $f(x)=y$. Thus $G\left(\tau_{x}\right)=\tau_{f(x)}$. It follows that $f$ is a path map with $\hat{f}=G$ if we show that $G(\sigma)(t)=f \sigma(t)$ for all $t \in I$. For each $t_{0} \in I$, we define $\rho_{t_{0}}: I \rightarrow I$ as the constant map into $t_{0}$. Then, since $G(\sigma) \rho=G(\sigma \rho)$, we get $G(\sigma)\left(t_{0}\right)=\left\{G(\sigma) \rho_{t_{0}}\right\}(s)=G\left(\sigma \rho_{t_{0}}\right)(s)=G\left\{\tau_{\sigma\left(t_{0}\right)}\right\}=f\left(\sigma\left(t_{0}\right)\right)$. Thus $\hat{f}=G$. Q.E.D.

REMARK. Theorem 3 is valid for arbitrary spaces $X$ and $Y$. Also, invariance under parametrization is a necessary condition for $G$ to be a $\hat{f}$. Finally, if $X$ is locally path connected, Hausdorff and 1st countable, and if $G$ is invariant under parametrization, then $G$ is continuous (in the $C-O$ topology).

We next consider the action of the functor $\pi^{I}$ (i.e., $\pi^{I}(X)=X^{I}$ ) from $T$ (topological spaces) to $S$ (sets). Let $\Upsilon$ be any subclass of $T$. We define the category $T(x)$ to contain all topological spaces as objects, but the morphisms of $T(x)$ are only those continuous maps that emanate from spaces of $x$. We also define the category $s(x)$ whose objects are $Z^{I}$, for $Z \in T$. The morphisms of $s(X)$ are the maps $G: X^{I} \rightarrow Y^{I}$ such that $x \in \mathcal{X}$, and $G$ is invariant under parametrization. If the spaces of $X$ are locally path connected, Hausdorff and 1st countable, then $\pi^{I}$ from $T(X)$ to $S(X)$ is not only faithful but invertible. Now we will state some necessary conditions on $X$ for $\pi^{I}$ to be invertible. 
Definition. The space $X$ is locally weakly path connected if for each $x_{0} \in X$ and each neighborhood $U$ containing $x_{0}$, there is an open set $V, x_{0} \in V \subseteq U$, such that for each $x \in V$, there is a path in $U$ connecting $x$ and $x_{0}$.

Theorem 4. If $X$ is a Hausdorff, 1st countable, space such that every path map is continuous, then $X$ is locally weakly path connected.

Proof. If $X$ is not locally weakly path connected, then there is an $x_{0} \in X$ and a neighborhood $U$ containing $x_{0}$, but $n o$ open set $V$ as in the definition above. Suppose $\left\{U_{n}\right\}$ is a neighborhood basis at $x_{0}$ with $U \supseteq U_{1} \supseteq U_{2} \supseteq \cdots$. We may choose $x_{n} \in U_{n}$ so that we have a sequence $x_{n} \rightarrow x_{0}$ with the property that no $x_{n}$ can be connected to $x_{0}$ by a path in $U$. Using this sequence $\left\{x_{n}\right\}$ we now define, for each open $U \subseteq X$, a new set $U^{\prime}$.

$$
\begin{aligned}
U^{\prime} & =U \quad \text { if } x_{0} \notin U \\
& =U \sim\left\{x_{1}, x_{2}, x_{3}, \cdots\right\} \quad \text { if } x_{0} \in U .
\end{aligned}
$$

Let $X^{\prime}$ be the space $X$ with the topology generated by $\left\{U^{\prime}\right\}$. We claim that the identity map $i: X \rightarrow X^{\prime}$ is a path map. To see this, we note that the local topology at $\bar{x}$ is the same in $X$ and $X^{\prime}$ if $\bar{x} \neq x_{0}$ because $X$ is Hausdorff. The only path $\sigma$ of $X$ that might not be a path of $X^{\prime}$ is an arc that intersects infinitely many $x_{i}^{\prime}$ 's. But if $\sigma$ is continuous, and $\sigma\left(t_{j}\right)=x_{j}$ for infinitely many $j$ 's, then there is a subsequence $\left\{t_{j_{k}}\right\}$ converging to a certain $t_{0}$. Further, $\sigma\left(t_{j_{k}}\right) \rightarrow \sigma\left(t_{0}\right)$. But $x_{j_{k}} \rightarrow x_{0}$ in $X$, and thus $x_{0}=\sigma\left(t_{0}\right)$. Now if $\sigma$ is a path, for any open $U^{\prime}$ about $x_{0}$, there is an $\epsilon$ such that $\sigma^{-1}\left(t_{0}-\epsilon, t_{0}+\epsilon\right) \subseteq U^{\prime}$. But this contradicts the selection of $x_{0}$ and $U$. Hence there is no such $\sigma$, and $i$ must be a path map. But $i$ is not continuous, contradicting the assumption that every path map $f: X \rightarrow Y$ is continuous. Therefore $X$ must be locally weakly path connected. Q.E.D.

We conclude with a brief treatment of the dual case:

TheOREM 5. If $Y$ is normal and its open sets are $F_{\sigma}$ 's, then every reverse path map $f: X \rightarrow Y$ is continuous.

Proof. Suppose $U$ is an open set of $Y$. There is a continuous map: $\phi: Y \rightarrow I$ such that $\phi(x)=1$ iff $x \in U$. (This follows from a strong version of Urysohn's lemma, using the hypothesis that open sets are $F_{\sigma}{ }^{\prime}$ s. $)^{2}$ Thus $f^{-1}(U)=(\phi f)^{-1}([0,1))$. But because $f$ is a reverse path map, $\phi f$ is continuous. Hence $f^{-1}(U)$ is open. Q.E.D.

2 James Dugundji, Topology, Allyn and Bacon, Boston, 1966, p. 148. 
Theorem 6. If $X$ and $Y$ are normal spaces and their open sets are $F_{\sigma}$ 's, and if $f: X \rightarrow Y$ is any function, then the following are equivalent:

(i) $f$ is a homeomorphism.

(ii) $f^{-1}$ exists, and $f$ and $f^{-1}$ are reverse path maps.

(iii) $I^{Y} \stackrel{f}{\rightarrow} I^{X}$ is a set theoretic isomorphism. (We define $\check{f}(\phi)=\phi f$.)

(iv) $f$ is a homeomorphism in the c-o topology.

REMARK. To pursue reverse path maps we could consider the action of $\pi_{I}$ (i.e., $\pi_{I}(X)=I^{X}$ ) and dualize the results for path maps.

UNIVERSITY OF ILLINOIS 